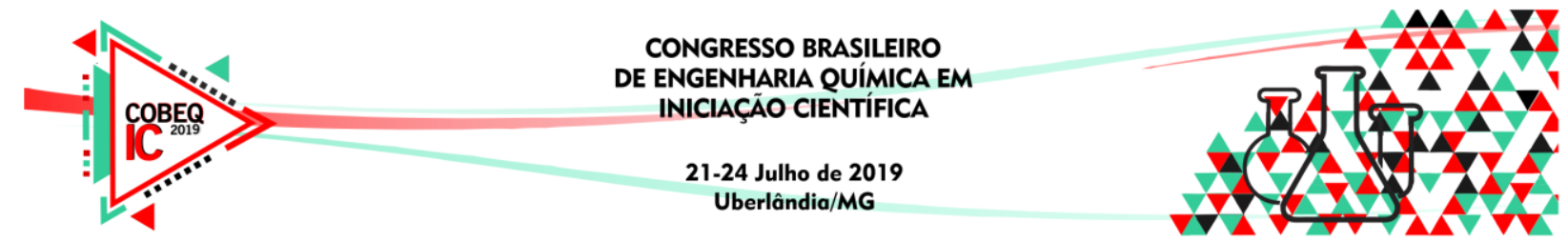

\title{
CARACTERIZAÇÃO QUÍMICA DA ÁGUA DE CHUVA COLETADA NO CENTRO DE DESENVOLVIMENTO DA TECNOLOGIA NUCLEAR-CDTN/CNEN
}

\author{
T. A. F. ALMEIDA ${ }^{1,2}$, H. E. L. PAMIERI ${ }^{1}$, L. M. L. A. AULER ${ }^{1}$, M. O. C. CARNEIRO ${ }^{1}$, F. \\ A. FORTES ${ }^{1}$ \\ ${ }^{1}$ Centro de Desenvolvimento da Tecnologia Nuclear - Comissão Nacional de Energia \\ Nuclear (CDTN/CNEN) \\ ${ }^{2}$ Centro Universitário Una - Campus Aimorés/ Belo Horizonte \\ E-mail para contato: thaisannie95@gmail.com
}

\begin{abstract}
RESUMO - Amostras de água de chuva mensais compostas coletadas no CDTN/CNEN durante o período de janeiro a dezembro de 2016 foram analisadas para os parâmetros $\mathrm{pH}$, condutividade elétrica, elementos maiores, menores e íons com o intuito de caracterizar estas amostras, como também avaliar as possíveis fontes emissoras dos constituintes das amostras. Os metais foram analisados pela técnica de Espectrometria de Massas por Plasma Indutivamente Acoplado (ICP-MS) e os íons pela Técnica de Cromatografia Líquida Iônica. Observou-se, em geral, que as mais altas concentrações dos parâmetros analisados ocorreram nas primeiras chuvas coletadas logo após o período seco e que os valores básicos de pH podem estar associados com a alta concentração de amônia nas águas de chuva, pois a amônia reage com os ácidos presentes na atmosfera formando os sais de amônio, que tornam as águas alcalinas. A análise dos coeficientes de correlação entre as espécies químicas quantificadas permitiu inferir que a incorporação de elementos nas águas de chuva provém de fontes naturais e antropogênicas. Como fonte antropogênica destaca-se o íon $\mathrm{NH}_{4}{ }^{+}$e o $\mathrm{Pb}$, como também o $\mathrm{SO}_{4}^{-}$e o $\mathrm{NO}_{3}{ }^{-}$, associados geralmente a emissões veiculares, industriais e queima de biomassa. Já os elementos $\mathrm{K}^{+}, \mathrm{Ca}, \mathrm{Na}, \mathrm{Si}, \mathrm{Mg}, \mathrm{Zn}, \mathrm{Cu}, \mathrm{Ba}, \mathrm{Mn}, \mathrm{Fe}, \mathrm{Al}, \mathrm{Sr}$ tem sua origem comum relacionados à ressuspensão de poeira do solo, construção civil e à intensa atividade de mineração na região de Belo Horizonte.
\end{abstract}

\section{INTRODUÇÃO}

Segundo o World Health Organization (WHO), a poluição atmosférica aumentou em 8\% dos anos de 2008 a 2013 e esta é responsável mais de sessenta mil mortes por ano no Brasil. Estes números evidenciam a importância de se analisar os poluentes na atmosfera, uma vez que a poluição em níveis muito altos pode ser tóxica a saúde.

A chuva faz parte do ciclo da água, que é o movimento contínuo da água que está presente nos oceanos, nos rios, nos açudes, nos continentes em geral e na atmosfera. Esse movimento é alimentado pela força da gravidade e pela energia do sol, que provocam a evaporação das águas dos oceanos e dos continentes. 


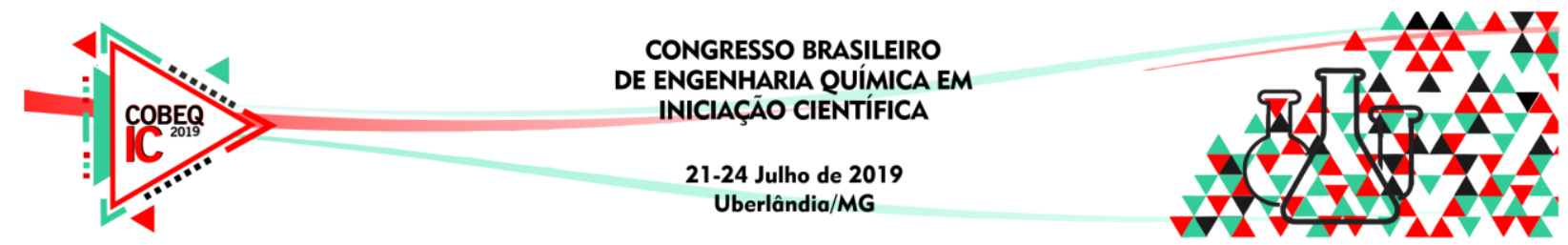

Portanto, percebe-se que as características químicas das águas pluviais são modificadas pela composição química da atmosfera que a circunda e estas características realizam um papel fundamental na indicação de poluentes solúveis na mesma. Sendo assim, a água de chuva pode ser usada como um índice da poluição do ar atmosférico e avaliar a sua composição é de suma importância para entender a origem das diferentes fontes de contribuição poluidora, sendo as fontes antropogênicas responsáveis por emitir a maior parte das substâncias potencialmente poluidoras (Xiao, 2016).

\section{OBJETIVO}

O objetivo do trabalho foi caracterizar a água de chuva mensal composta da estação do CDTN/CNEN dos meses de janeiro a dezembro do ano de 2016 para os seguintes parâmetros: $\mathrm{Al}, \mathrm{Cr}, \mathrm{Mn}, \mathrm{Fe}, \mathrm{Ca}, \mathrm{Mg}, \mathrm{Ni}, \mathrm{Cu}, \mathrm{Zn}, \mathrm{As}, \mathrm{Cd}, \mathrm{Ba}, \mathrm{Pb}, \mathrm{Rb}, \mathrm{Sr}$ e Sb; os cátions $\mathrm{Na}^{+}, \mathrm{NH}_{4}{ }^{+}$e $\mathrm{K}^{+}$, os ânions $\mathrm{F}^{-}, \mathrm{Cl}^{-}, \mathrm{NO}_{2}^{-}, \mathrm{NO}_{3}{ }^{-}$e $\mathrm{SO}_{4}^{-}, \mathrm{pH}$, condutividade elétrica e a identificação das possíveis fontes emissoras dos constituintes das amostras.

\section{METODOLOGIA}

As amostras da água de chuva foram coletadas mensalmente na estação do CDTN durante o ano de 2016 nos meses de: janeiro, fevereiro, março, junho, setembro, outubro, novembro e dezembro.

O procedimento para a coleta das amostras de precipitação do CDTN seguiu o padrão Ville de Paris devido ao clima tropical da cidade de Belo Horizonte. O pluviômetro foi instalado seguindo os procedimentos padrões deste equipamento. Para o armazenamento das águas de chuva foram utilizados contêineres de polietileno, armazenados em caixas térmicas para evitar o contato solar direto (Cota et al, 2011).

Após a coleta, as amostras foram filtradas por um filtro de 0,45 $\mu \mathrm{m}$ para análise posterior. As análises para $\mathrm{Al}, \mathrm{Cr}, \mathrm{Mn}, \mathrm{Fe}, \mathrm{Ca}, \mathrm{Mg}, \mathrm{Ni}, \mathrm{Cu}, \mathrm{Zn}, \mathrm{As}, \mathrm{Cd}, \mathrm{Ba}, \mathrm{Pb}, \mathrm{Rb}, \mathrm{Sr}$ e Sb foram realizadas usando o Espectrômetro de Massas com Plasma Indutivamente Acoplado (ICP-MS) Elan DRC-e Axial Field da Perkin Elmer. Os íons $\mathrm{Na}^{+}, \mathrm{NH}_{4}^{+} \mathrm{K}^{+}, \mathrm{F}^{-}, \mathrm{Cl}^{-}, \mathrm{NO}_{2}^{-}$, $\mathrm{NO}_{3}{ }^{-}$e $\mathrm{SO}_{4}{ }^{-}$foram analisados pela técnica de Cromatografia Líquida usando o Cromatógrafo Shimadzu LC-10AD VP. A condutividade elétrica das amostras foi medida pelo condutivímetro 712 da Metrohm e o pH pelo medidor de pH 744 também da marca Metrohm.

\section{RESULTADOS E DISCUSSÕES}

\section{1. pH e Condutividade elétrica (CE)}

A Tabela 1 dispõe os resultados obtidos para o $\mathrm{pH}$ e a $\mathrm{CE}$ das amostras de água de chuva compostas coletadas. Os valores de $\mathrm{pH}$ variaram de 7,51 a 8,31 sendo o mês de fevereiro com o valor mais alto, e o mês de março com o valor mais baixo.

Os resultados destas amostras mostraram ter caráter básico, não caracterizando uma chuva ácida, já que para ser considerada ácida ela deve apresentar valores de pH inferiores a 5,65 (Jesus, 1996). 


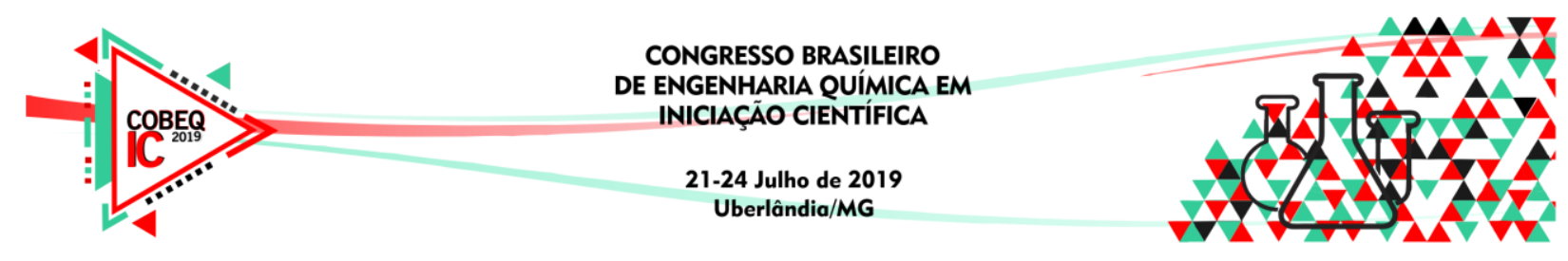

Tabela 1. Valores de pH e condutividade elétrica das amostras mensais compostas

\begin{tabular}{|c|c|c|c|c|c|c|c|c|}
\hline Mês & Jan & Fev & Mar & Jun & Set & Out & Nov & Dez \\
\hline pH & 7,99 & 8,31 & 7,29 & 7,87 & 8,06 & 7,81 & 7,53 & 8,09 \\
\hline CE $(\boldsymbol{\mu S} / \mathbf{c m})$ & 5,67 & 11,47 & 58,44 & 97,35 & 171 & 64,45 & 38 & 137 \\
\hline
\end{tabular}

As Figuras. 1 e 2 mostram os gráficos dos valores da $\mathrm{CE}$ e $\mathrm{pH}$. Como a condutividade elétrica expressa a concentração de sais dissolvidos, isto possibilita que este parâmetro seja utilizado como indicador para a poluição atmosférica (Andrade $e$ tal, 2017). Percebe-se que nos meses de setembro e dezembro ocorreram os picos mais altos de condutividade devido à alta concentração de íons nestes meses.

Figuras 1 e 2 - Gráficos de condutividade e pH da água de chuva de 2016
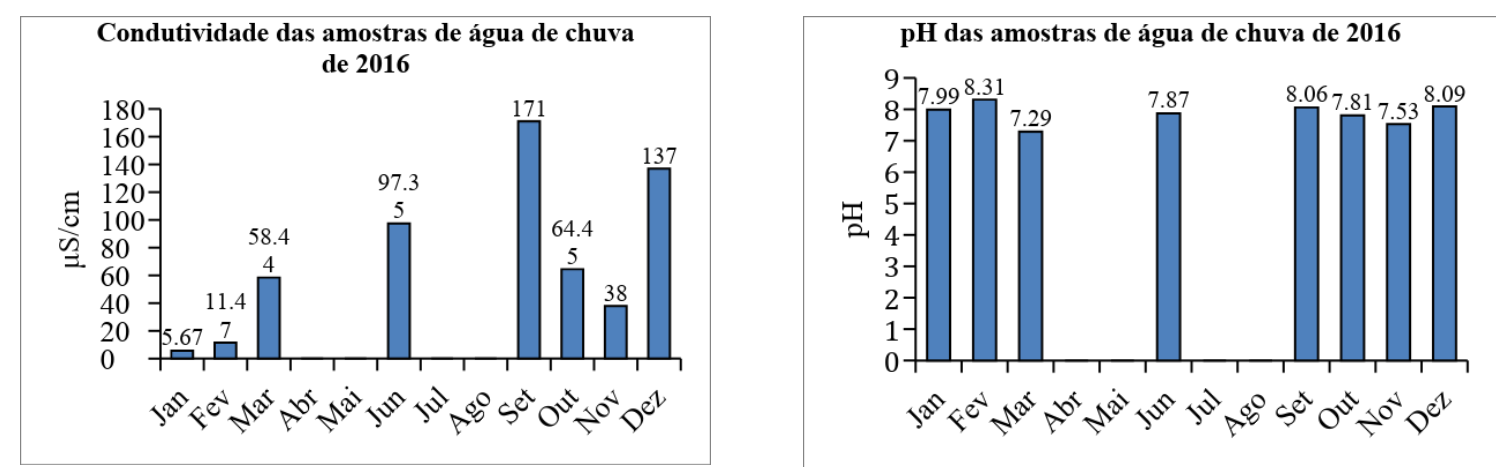

\section{2. Íons Principais e os Metais}

A Tabela 2 apresenta os valores das concentrações dos íons principais nas amostras de água de chuva do ano de 2016. Nestas amostras, o íon que se destacou foi o amônio. Tal cátion normalmente é um subproduto da decomposição dos fertilizantes nitrogenados utilizados na agroindústria (Coelho et al, s.d.), e também pode ter origem de atividade veicular (Silva, 2015).

A Tabela 3 mostra as concentrações dos elementos metálicos e os respectivos coeficientes de variação (CV) em porcentagem, das amostras compostas de água de chuva, determinados pela técnica de Espectrometria de Massas (ICP-MS) e na Figura 3 o gráfico correspondente destes elementos nestas mesmas amostras. 


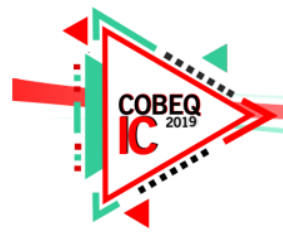

Tabela 2 - Concentração dos cátions e ânions em amostras de águas de chuva/2016

\begin{tabular}{|c|c|c|c|c|c|c|c|c|}
\hline Mês & $\begin{array}{c}\mathrm{Na}^{+} \\
\left(\mathrm{mg} \cdot \mathrm{L}^{-1}\right)\end{array}$ & $\begin{array}{c}\mathrm{NH}_{4}^{+} \\
\left(\mathrm{mg} \cdot \mathrm{L}^{-1}\right)\end{array}$ & $\begin{array}{c}\mathrm{K}^{+} \\
\left(\mathrm{mg} \cdot \mathrm{L}^{-1}\right)\end{array}$ & $\begin{array}{c}\mathrm{F}^{-} \\
\left(\mathrm{mg} \cdot \mathrm{L}^{-1}\right)\end{array}$ & $\begin{array}{c}\mathrm{Cl}^{-} \\
\left(\mathrm{mg} \cdot \mathrm{L}^{-1}\right)\end{array}$ & $\begin{array}{c}\mathrm{NO}_{2}^{-} \\
\left(\mathrm{mg} \cdot \mathrm{L}^{-1}\right)\end{array}$ & $\begin{array}{c}\mathrm{NO}_{3}^{-} \\
\left({\mathrm{mg} . \mathrm{L}^{-1}}^{-}\right.\end{array}$ & $\begin{array}{c}\mathrm{SO}_{4}^{-} \\
\left(\mathrm{mg} \cdot \mathrm{L}^{-1}\right)\end{array}$ \\
\hline Jan & $0,60 \pm 0,04$ & $33,5 \pm 0,60$ & $1,1 \pm 0,20$ & $0,22 \pm 0,02$ & $0,32 \pm 0,01$ & $<0,05$ & $0,57 \pm 0,02$ & $0,48 \pm 0,02$ \\
\hline Fev & $0,90 \pm 0,10$ & $46,5 \pm 0,30$ & $2,1 \pm 0,10$ & $0,17 \pm 0,01$ & $0,51 \pm 0,01$ & $<0,05$ & $1,06 \pm 0,04$ & $0,66 \pm 0,03$ \\
\hline Mar & $0,35 \pm 0,01$ & $13,0 \pm 0,10$ & $1,4 \pm 0,10$ & $0,05 \pm 0,01$ & $0,32 \pm 0,01$ & $<0,05$ & $0,56 \pm 0,02$ & $1,50 \pm 0,10$ \\
\hline Jun & $0,83 \pm 0,01$ & $18,3 \pm 0,10$ & $1,7 \pm 0,20$ & $<0,05$ & $0,43 \pm 0,01$ & $0,08 \pm 0,01$ & $1,50 \pm 0,10$ & $3,00 \pm 0,10$ \\
\hline Set & $2,00 \pm 0,10$ & $25,4 \pm 0,10$ & $4,0 \pm 0,10$ & $<0,05$ & $2,50 \pm 0,02$ & $<0,05$ & $2,00 \pm 0,10$ & $7,00 \pm 0,30$ \\
\hline Out & $0,34 \pm 0,05$ & $12,2 \pm 0,10$ & $1,1 \pm 0,20$ & $<0,05$ & $0,40 \pm 0,01$ & $<0,05$ & $0,86 \pm 0,03$ & $1,10 \pm 0,05$ \\
\hline Nov & $1,1 \pm 0,10$ & $6,3 \pm 0,10$ & $2,6 \pm 0,40$ & $<0,05$ & $0,41 \pm 0,01$ & $<0,05$ & $<0,05$ & $0,57 \pm 0,03$ \\
\hline Dez & $0,81 \pm 0,02$ & $27,0 \pm 0,30$ & $0,6 \pm 0,10$ & $<0,05$ & $0,16 \pm 0,01$ & $<0,05$ & $0,31 \pm 0,01$ & $0,30 \pm 0,01$ \\
\hline
\end{tabular}

Tabela 3 - Concentração dos elementos metálicos nas amostras mensais compostas

\begin{tabular}{|c|c|c|c|c|c|c|c|c|c|c|c|c|c|c|c|c|c|}
\hline \multicolumn{2}{|c|}{ Analito } & \multicolumn{2}{|c|}{ Janeiro } & \multicolumn{2}{|c|}{ Fevereiro } & \multicolumn{2}{|c|}{ Março } & \multicolumn{2}{|c|}{ Junho } & \multicolumn{2}{|c|}{ Setembro } & \multicolumn{2}{|c|}{ Outubro } & \multicolumn{2}{|c|}{ Novembro } & \multicolumn{2}{|c|}{ Dezembro } \\
\hline $\mathrm{Ca}(\mu g \mathrm{~g})$ & e CV(\%) & 333 & 1 & 652 & 2 & 640 & 1 & 1261 & 1 & 3836 & 1 & 717 & 1 & 472 & 2 & 53 & 5 \\
\hline $\mathrm{Mg}(\mu \mathrm{g} / \mathrm{L})$ & e CV(\%) & 23 & 3 & 79 & 2 & 76 & 3 & 196 & 2 & 673 & 2 & 86 & 1 & 101 & 1 & $\ll 4$ & . \\
\hline $\mathbf{S}(\mu g \mathrm{~L})$ & e CV(\%) & 163 & 1 & 173 & 13 & 241 & 2 & 174 & 10 & 629 & 6 & 20,5 & 4 & 104 & 2 & 80,1 & 2 \\
\hline $\operatorname{Al}(\mu g g)$ & e CV(\%) & 2,61 & 4 & 19 & 4 & 5,25 & 1 & 9,98 & 1 & 24,5 & 1 & 40,4 & . & 8,7 & 2 & 2,52 & 1 \\
\hline $\mathrm{Cr}\left(\mu g^{\prime} \mathrm{L}\right)$ & $\mathrm{eCV}(\%)$ & $<1,4$ & $\cdot$ & $<1,4$ & - & $<1,4$ & - & $<1,4$ & $\cdot$ & 2,39 & 1 & $<1,4$ & - & $<1,4$ & - & $<1,4$ & - \\
\hline $\operatorname{Mn}(\mu \mathrm{g} / \mathrm{L})$ & $\mathrm{eCV}(\%)$ & 4,72 & 2 & 9 & 1 & 9,56 & 1 & 16 & 1 & 64,6 & 2 & 8,4 & 1 & 10,8 & 1 & 2,4 & 1 \\
\hline $\mathrm{Fe}(\mu \mathrm{g} \mathrm{L})$ & $\mathrm{eCV}(\%)$ & 8 &. & 21 & 2 & 8,38 & 1 & 12,9 & 1 & 28,7 & 3 & $<8$ & $\cdot$ & 14,8 & 1 & $<8$ & . \\
\hline $\mathrm{Ni}(\mu g \mathrm{~g} L)$ & e CV(\%) & $<0,6$ & $\cdot$ & $<0,6$ & $\cdot$ & $\$ 0,6$ & $\cdot$ & 1,02 & 1 & 1,29 & 1 & $<0,6$ & $\cdot$ & 1,52 & 1 & 4,6 & $\cdot$ \\
\hline $\mathrm{Cu}(\mu g \mathrm{~g})$ & e CV(\%) & 23,1 & 2 & 71,6 & 25 & 38,5 & 5 & 64,6 & 8 & 112 & 3 & 20,8 & 10 & 59,6 & 8 & 37,6 & 9 \\
\hline $\operatorname{Zn}(\mu \mathrm{g} L)$ & $\mathrm{e} \mathrm{Cl}(\%)$ & 84,4 & 2 & 89,2 & 45 & 149 & 10 & 209 & 7 & 147 & 3 & 132 & 10 & 128 & 11 & 77,6 & 12 \\
\hline $\operatorname{As}(\mu g g)$ & e CV(\%) & $<0,1$ & . & 0,07 & 4 & 0,10 & 1 & 0,20 & 1 & 0,297 & 1 & 0,10 & 1 & 0,087 & 1 & 40,08 & $\cdot$ \\
\hline $\mathrm{Cd}(\mu g \mathrm{~g} L)$ & e CV(\%) & 0,06 & & 0,12 & 1 & 0,081 & 4 & 0,10 & 1 & 0,09 & 1 & 0,08 & 1 & 0,085 & 1 & 0,054 & 1 \\
\hline $\mathrm{Ba}(\mu g \mathrm{~g})$ & e CV(\%) & 20,1 & 2 & 13,4 & 1 & 12,1 & 1 & 12,10 & 1 & 31,90 & 2 & 20,2 & 1 & 17 & 1 & 5,27 & 1 \\
\hline $\mathrm{Pb}(\mu g \mathrm{~L})$ & $\mathrm{eCl}(\%)$ & 76,7 & 2 & 169 & 19 & 176 & 5 & 193 & 5 & 70,20 & 3 & 65,2 & 9 & 156 & 5 & 250 & 6 \\
\hline $\mathrm{Rb}(\mu g \mathrm{~L})$ & $\mathrm{e} \mathrm{CV}(\%)$ & 0,71 & 2 & 2,61 & 1 & 1,01 & 1 & 1,22 & 1 & 7,58 & 1 & 0,68 & 1 & 2,35 & 1 & 0,18 & 2 \\
\hline$\$(\mu g \mathrm{~L})$ & e CV(\%) & 2,65 & 1 & 5,51 & 1 & 5,09 & 1 & 11,30 & 1 & 31,0 & 1 & 6,02 & 1 & 4,54 & 1 & 1,26 & 1 \\
\hline$s b(\mu g / L)$ & e CV(\%) & 0,084 & 1 & 0,18 & 1 & 0,13 & 1 & 0,16 & 3 & 0,28 & 2 & 0,087 & 5 & 0,15 & 1 & 0,04 & 10 \\
\hline
\end{tabular}

Na Figura 3 é perceptível a relevância do magnésio e do silício no mês de setembro, destacando dos demais elementos. Para Xiao (2016) a principal fonte para tais elementos é da poeira que é levantada devido às construções civis e a própria ressuspensão de micropartículas presentes nos solos.

O cálcio também se encontra com um valor elevado, sendo este elemento similar ao magnésio e possuindo também as mesmas fontes. $\mathrm{O} \mathrm{Pb}$ encontrado em todas as amostras é 


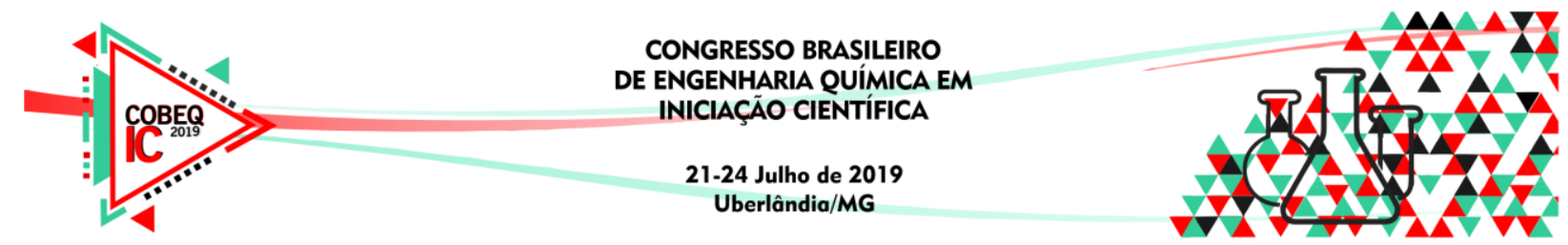

reconhecidamente tóxico à população exposta e pode ter origem natural como antropogênica, sendo a sua presença mais significativa em atmosferas urbanas (Xiao, 2016).

Figura 3- Gráfico dos elementos químicos encontrados nas amostras mensais compostas de água de chuva do ano de 2016

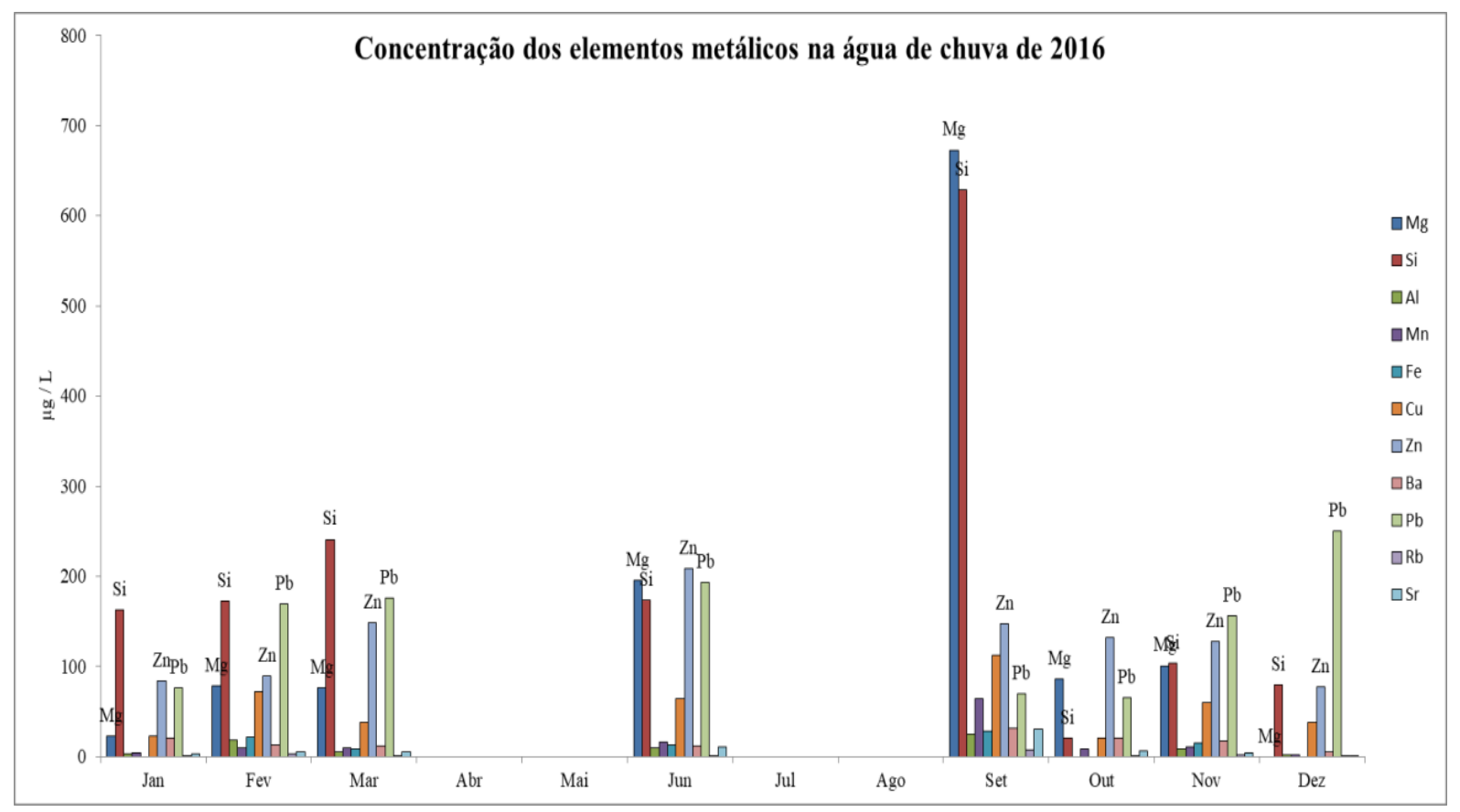

\section{CONCLUSÕES}

Pode-se concluir que os valores básicos de $\mathrm{pH}$ podem estar associados com a alta concentração de amônia nas águas de chuva, pois a amônia reage com os ácidos presentes na atmosfera formando os sais de amônio, que tornam as águas alcalinas (Felix et al, 2004; Manahan, 2013). O amônio, assim como os nitratos e os sulfatos geralmente estão associados a fontes antropogênicas, tais como as atividades industriais e a emissão de poluentes de carburadores dos veículos $\mathrm{A}$ amônia também pode estar relacionada à queima de biomassa (atividades agrícolas e incêndios florestais) (Hu et al, 2003).

Observa-se também que o mês de setembro foi o mês em que houve o maior pico de condutividade, e concentrações dos íons e elementos, em especial o cálcio e magnésio. Estas elevadas concentrações podem ser atribuídas ao acúmulo de substâncias na atmosfera durante períodos de julho e agosto. Segundo $\mathrm{Hu}$ et al (2003), estas substâncias podem estar relacionadas com a queima de biomassa e a suspensão de poeira terrestre.

A análise dos coeficientes de correlação entre as espécies químicas quantificadas permitiu inferir que a incorporação de elementos nas águas de chuva provém de fontes naturais e antropogênicas. Como fonte antropogênica destaca-se o íon $\mathrm{NH}_{4}{ }^{+}$e o $\mathrm{Pb}$, como 


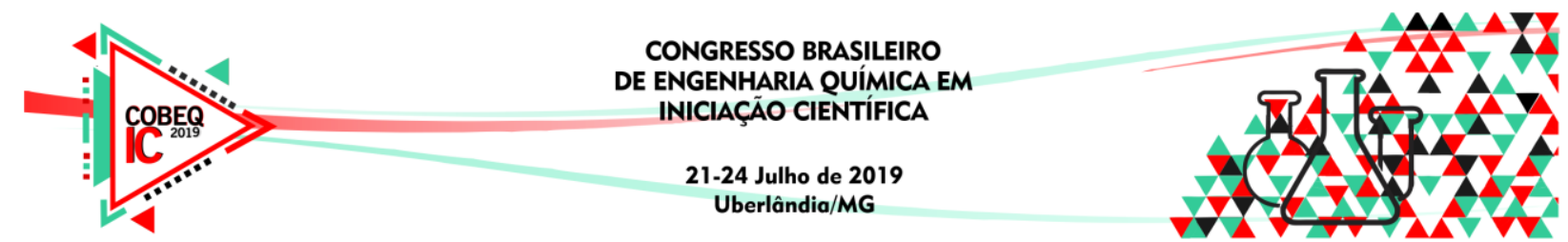

também o $\mathrm{SO}_{4}{ }^{-}$e o $\mathrm{NO}_{3}{ }^{-}$, associados geralmente a emissões veiculares, industriais e queima de biomassa. Já os elementos $\mathrm{K}^{+}, \mathrm{Ca}, \mathrm{Na}, \mathrm{Si}, \mathrm{Mg}, \mathrm{Zn}, \mathrm{Cu}, \mathrm{Ba}, \mathrm{Mn}, \mathrm{Fe}, \mathrm{Al}, \mathrm{Sr}$ tem sua origem comum relacionados à ressuspensão de poeira do solo, construção civil e à intensa atividade de mineração na região de Belo Horizonte.

\section{REFERÊNCIAS BIBLIOGRÁFICAS}

Andrade, M. C. S. S., Andrade, L. R. S., Curi, R. C. "Análise de pH e condutividade elétrica como indicadores de poluição atmosférica de chuva de Campina Grande - PB." III Workshop Internacional sobre Água no Semiárido Brasileiro, Universidade Federal de Campina Grande, 2017.

Assunção, A. L. C. "Hidroquímica e qualidade das águas subterrâneas do sistema aquífero Dunas-Barreiras na região de Parnamirim, $R N$ ", Dissertação para o curso de pós-graduação em geodinâmica e geofísica (PPGG), UFRN, Natal, RN, Dezembro de 2016.

Brasil (Ministério do Meio Ambiente), 2007, "Cíclo Hidrológico", em: < http://www.mma.gov.br/agua/recursos-hidricos/aguas-subterraneas/ciclo-hidrologico $>$, acessado em Abril de 2018.

Coelho, A. M., e tal. "Eficiência Agronômica do Cloreto de Amônio e Bicarbonato de Amônio Como Fontes de Nitrogênio Para a Cultura do Milho." Embrapa Milho e Sorgo, Sete Lagoas, MG; CP 151, s. d.

Cota, S. et al." Environmental isotopes and chemistry Characterization of the rainfall at the cdtn gnip Station", International Nuclear Atlantic Conference - INAC 2011, Belo Horizonte,MG,2011.

Felix, E. P.; Cardoso, A. A. "Amônia (NH3) atmosférica: fontes, transformação, sorvedouros e métodos de análise.” Química Nova, v. 27, n. 1, São Paulo, jan./fev. 2004.

Jesus, E. F. R. "A importância do estudo das chuvas ácidas no contexto da abordagem climatológica”, Sitientibus, Feira de Santana, n.14, pp.143-153, 1996.

Hu. G, P., Balasubramian, R., Wu. C. "Chemical Characterization of Rainwater Constituents at Singapore." Chemosphere, n 51, pp. 747-755, 2003.

Manahan, S. E. “Química Ambiental.” 9a edição. Ed. Bookman, Porto Alegre, pp. 944, 2013.

WHO (World Health Organization), 2016, "The air pollution in Quatis, Brazil", em:

< http://breathelife2030.org/city-data-page/?city=196 > , acessado em Abril de 2018.

Xiao, J. "Chemical Composition and Source Identification of Rainwater Constituents at an Urban Site in Xi'an”. Environ Earth Sci. pp.13, 2016. 